\title{
Tracer gas experiments in subways using an integrated measuring and analysis system for sulphur hexafluoride
}

\author{
Markus Brüne ${ }^{1}$, Jonathan Spiegel ${ }^{1}$, Karin Potje-Kamloth ${ }^{2}$, Christian Stein ${ }^{3}$, Andreas Pflitsch ${ }^{1}$ \\ ${ }^{1}$ Ruhr-University Bochum, Universitätsstraße 150, 44801 Bochum, Germany \\ ${ }^{2}$ Fraunhofer ICT-IMM, Carl-Zeiss-Straße 18-20, 55129 Mainz, Germany \\ ${ }^{3}$ smartGAS Mikrosensorik GmbH, Kreuzenstraße 98, 74076 Heilbronn, Germany
}

\begin{abstract}
:
Several sulphur hexafluoride $\left(\mathrm{SF}_{6}\right)$ tracer gas experiments were conducted in a subway system to empirical measure the possible pathways of toxic gas for subway tunnels and stations. A new mobile integrated measuring and analysis system was used, achieving high sample rates and a long measurement time. Due to the mobility of the sensor, tracer gas experiments were also carried out inside running subway coaches. All experiments showed a common pattern: The pathways of tracer gas dispersion often overlaps with escape routes, and these were contaminated within a few minutes. In case of catastrophic circumstances, like terrorist attacks or subway fires, some escape routes will became deathly traps, but the results also showed that others are safe. With the new sensor technique it will be possible to make safety assessments for escape routes in underground transportation facilities.
\end{abstract}

Key words: sulphur hexafluoride, gas sensor, integrated analysis and measuring system, tracer gas experiments, propagation paths of toxic airborne substances

\section{Introduction and Motivation}

The results of tracer gas experiments help to understand the possible spread of toxic airborne substances for example in subway stations, which are vulnerable in the face of terrorism. In contrast to the period of 1982-91, where deliberate acts of malice caused 1,327 deaths among air travellers and none among subway commuters, the pattern reserved between 2002 and 2011 [1]. As hindering terrorists from entering subway stations is very difficult, preparedness and responsiveness are very important. This includes, that the pathways of airborne toxic substances should be known and do not in any case overlap with emergency escape routes in subway stations.

Sulphur hexafluoride $\left(\mathrm{SF}_{6}\right)$ has become an accepted standard in underground ventilation studies [2]. In the past, the contamination of air with SF6 was often determined by manual air probes with $60 \mathrm{ml}$ syringes and subsequent analysis by a gas chromatograph. This method has some obvious disadvantages: The number of syringes is limited to the laboratory capacity, which results in a short measurement time and a low sample rate. Notably, the time period between taking and analyzing the samples could pollute the probes. The involvement of several persons is also a source of error. The development of a mobile, battery powered, integrated measuring and analysis system for $\mathrm{SF}_{6}$, recording with a sample rate of two seconds, solves these disadvantages.

\section{Tracer gas experiments}

In February 2014 several tracer gas experiments were conducted in the subway system of a major European city. The objectives of these experiments were:

- Determine pathways of gas dispersion inside subway stations

- Determine the influence of train traffic on the pathways of gas dispersion

- Determine the affection of a whole subway system by releasing tracer gas inside running subway coaches

\section{The experimental site: The subway system}

The experiments were carried out inside the underground station of the subway of the major European city. The Underground network consists basically of two lines, running north to south an east to west with a total length of about $4 \mathrm{~km}$. Due to the orography, the tunnel of the east-west line ascends rom East Station to West Station by $15 \mathrm{~m}$ in elevation while the 
north-south line rises around $30 \mathrm{~m}$ towards North Station (see Fig. 2). Both lines cross at Central Station (see Fig. 1 \& 3).

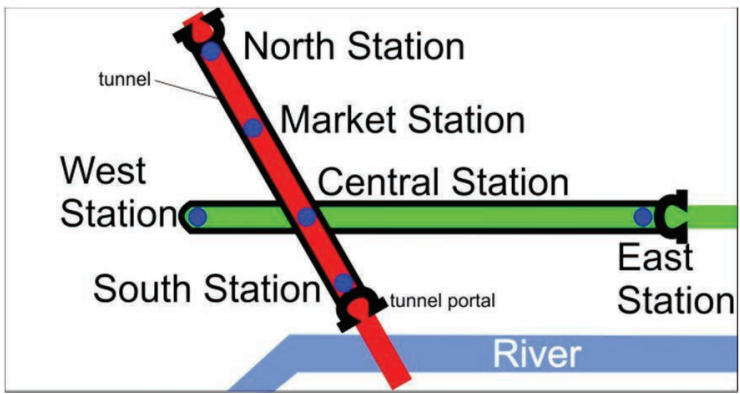

Fig. 1: Overview of underground stations of the subway system

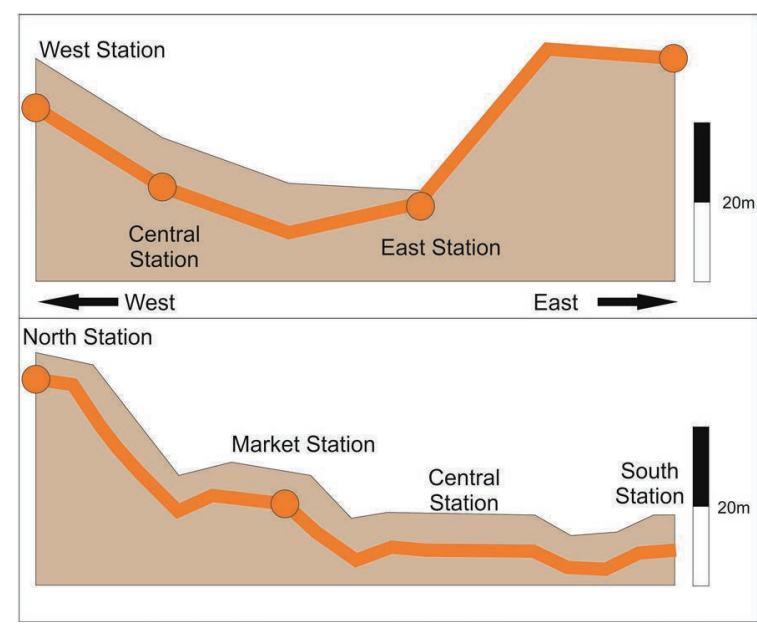

Fig. 2: Cross section of the west-east line (top) and north-south line (bottom)

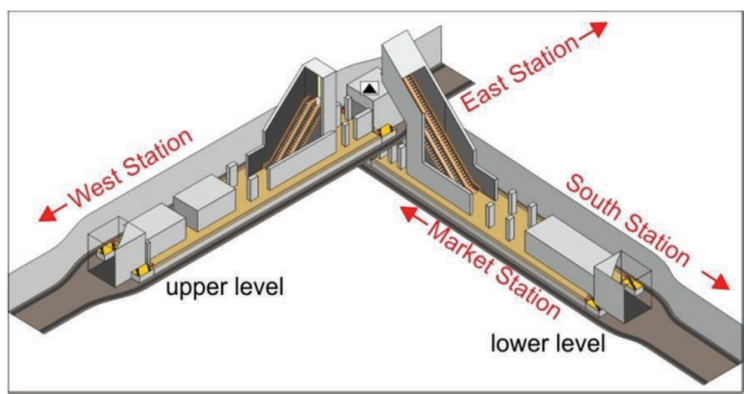

Fig. 3: 3-dimensional view of Central Station

\section{The Gas Sensor}

In contrast to former experiments, which reflect only rough pictures of the gas dispersion [3], the new mobile integrated measuring and analysis system can provide much more detailed data [4]. As the sensor platform is a battery powered hand-held device, more experiment settings are possible (see Fig. 4). The device can be connected to a temperature sensor and an ultra-sonic anemometer to record necessary parameters to provide boundary conditions for numerical simulations (CFD).
The tracer gas data can be used to validate dispersion forecasts of numerical simulations with empirical data. The heart of the device is a cuvette where laser diodes emitting a very narrow band of light wavelength in the range of the absorbance of $\mathrm{SF}_{6}$ at $10.7 \mu \mathrm{m}$.

\section{The Influence of train traffic}

During the night of February $22^{\text {nd }}$, two tracer gas experiments were conducted to determine the gas propagation for a station and its adjacent tunnels and stations. Central Station was selected because of its two platform levels which each belong to one of the two subway lines. The experiment was done twice. The first gas release was done during the operational time of the subway at 20:15 UTC. An amount of $2.15 \mathrm{~kg} \mathrm{SF}_{6}$ was released during ten minutes. In order to compare traffic times with no train movement, the experiment was repeated in the same night. At 00:52 UTC $2.06 \mathrm{~kg}$ of $\mathrm{SF}_{6}$ was released for about eight minutes during operational break.

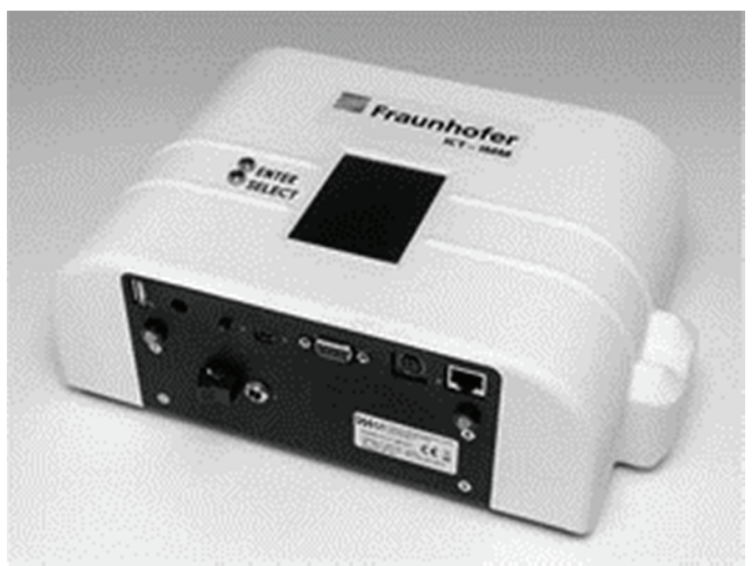

Fig. 4: Integrated analysis and measuring system

The upper level was contaminated within three minutes during the train traffic experiment whereas it takes only two minutes longer without traffic. This confirms an experiment carried out one day earlier [5], which focused only on Central Station. The maximum recorded tracer gas concentration was higher and more parts of the platform levels were affected with train traffic. The propagation paths were wider distributed due to several train movements (see Fig. 5).

When looking at the adjacent stations a similar pattern can be observed. The maximum recorded concentration was found at West Station during the operational break. During the operational time the sensor (19) detected first values after $17 \mathrm{~m} 30 \mathrm{~s}$ sensor (20) followed 21 minutes after the gas release. But the first measured concentration was on a very low level. 


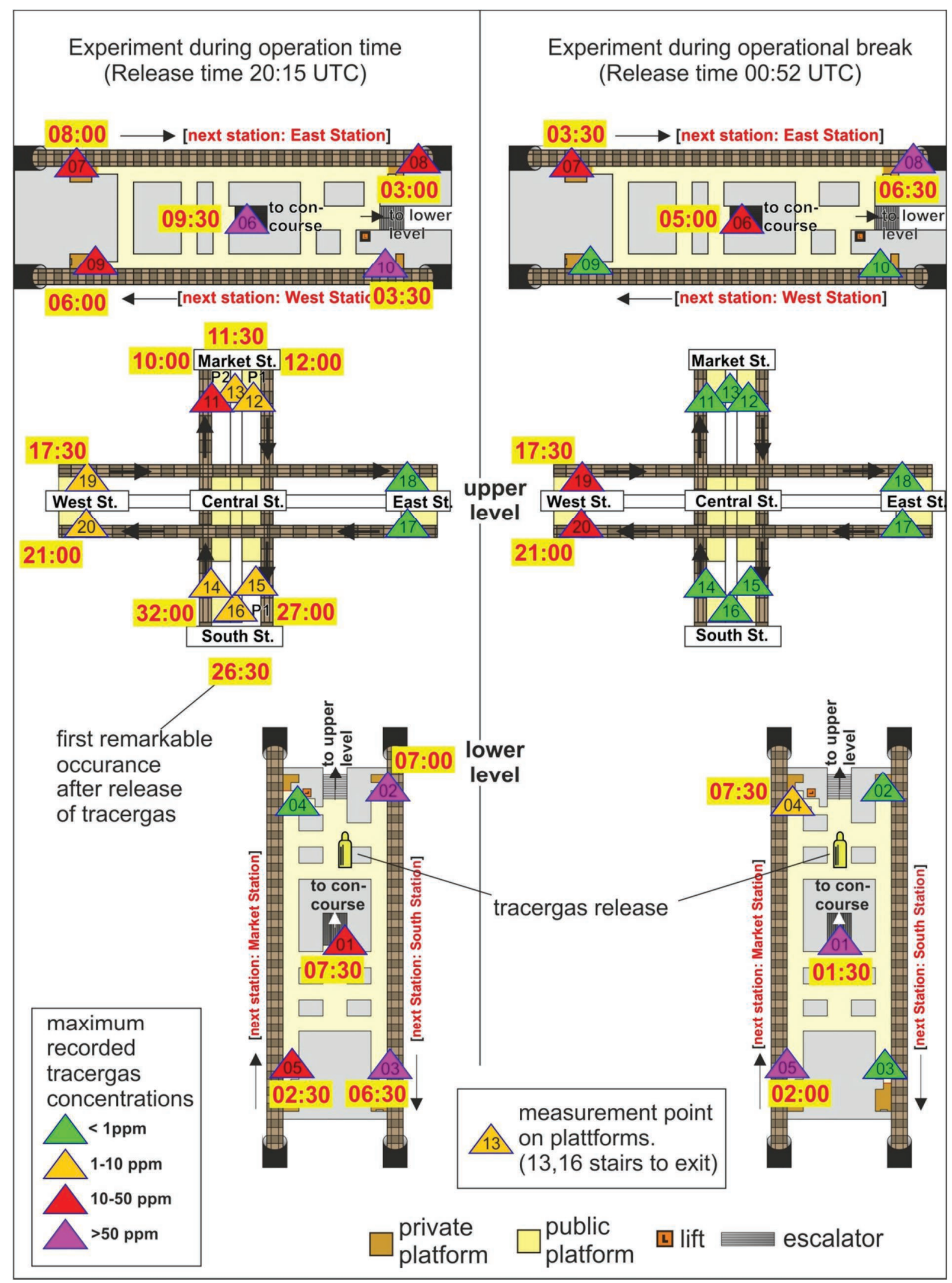

Fig. 5. Comparison of tracer gas experiments during traffic times (left) and operational break (right) 


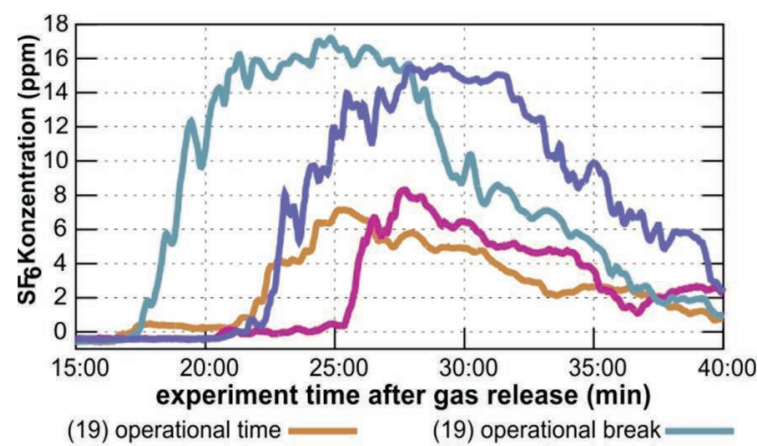

(20) operational time $\quad$ (20) operational break

Fig. 6: $S F_{6}$ concentrations at station West Station. The numbers in brackets contribute to the measurement points (see Fig. 4)

As around the same time a subway train reaches West Station from Central Station. It can be assumed that this first detected concentration is caused by train movements.

A steeper rise of values occurred approximately five minutes later. In contrast, during operational break, this steep ascent coincide with the first remarkable increase of concentration (see Fig. 6)

Market and South Station were only affected during the experiment with traffic. Due to the ascending tunnel from South Station to North Station a natural background air flow, caused by a buoyancy, effect is established.

In fact the movement of the running trains disturb the background air flow. Regardless the tracer gas reaches the Market station significantly earlier $(10 \mathrm{~min})$ than the South Station (26 min).

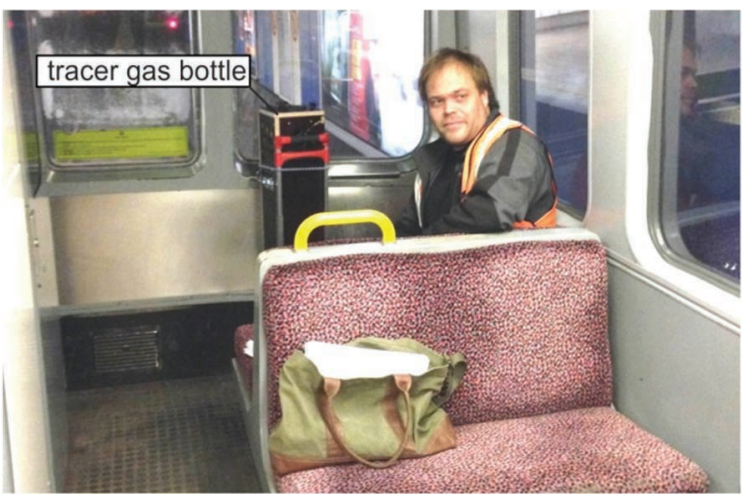

Fig. 7: Gas release inside a subway train

\section{Tracer gas release inside a subway train}

The hand-held integrated analysis and measuring system offered the possibility to measure tracer gas inside operating trains. An $\mathrm{SF}_{6}$ bottle was located in the back coach of a two wagon subway train (see Fig. 7). Two minutes before the trains reaches Central Station, the tracer gas was released inside the back coach continuously with a relative low flow rate.

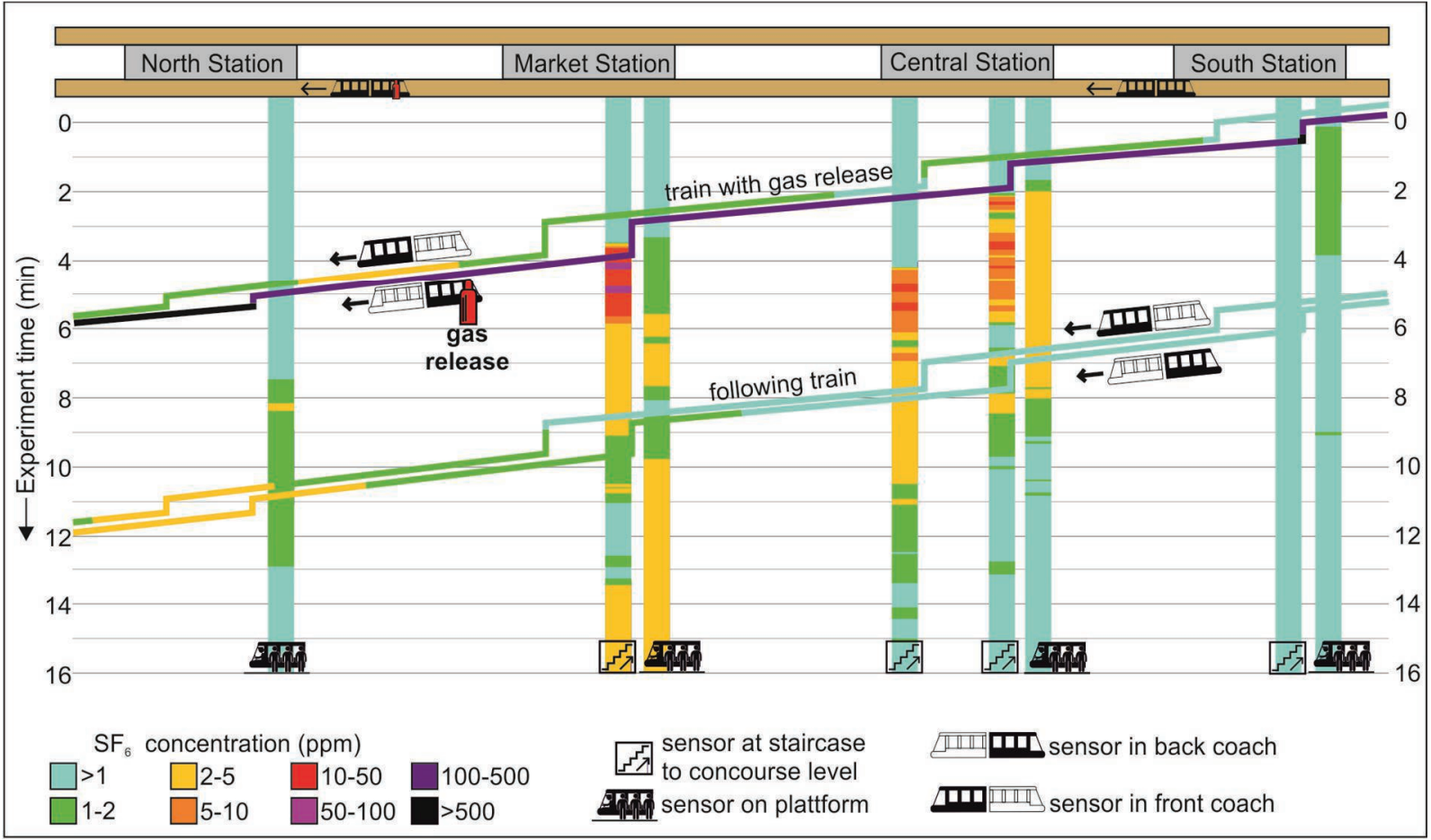

Fig. 8: Tracer gas release inside a running train in the back coach. The lines represent the measured concentration while the train is running. The bars represent sensors on the platforms inside the station. 
The release was stopped seven minutes later when the doors closed at North Station. By then an amount of $1.49 \mathrm{~kg}$ was let out of the gas bottle. The contaminated coach reached very high values of $800 \mathrm{ppm}$ for a period of two minutes. The threshold limit of $\mathrm{SF}_{6}$ is $1,000 \mathrm{ppm}$ for an average working day of eight hours. The chosen amount of released tracer gas in such experiments provides sufficient detailed data without harming the passengers.

Shortly after the train calls at a station and the doors opened, a significant rise of tracer gas concentration was observed at the subway stations. At Central and Market Station the contamination was an addition of the outflow of the coach and the propagation of gas from the tunnels, mainly caused by the suction of the train due to the piston effect.

Moreover, the highest concentration inside the station was quickly found in the staircases to the concourse levels and the exits. This is a further verification that staircases in subway stations are sucking air from the lower parts of the structure.

In the front section of the train, which is separated from the back, a small rise of $\mathrm{SF}_{6}$ concentration was observed. At the third stop more significant higher values were logged.

Further gas sensors were also located in the following train. Contaminated air from the subway station flows in the coaches, while the doors are opened during train stop. The concentration accumulates up to $3 \mathrm{ppm}$ after the passage of Market Station (see Fig. 8).

\section{Main results of tracer gas experiments}

In general, subway stations are mostly over warmed. The deeper a station is in the ground a fortiori long connections to the surface (stairway, escalator) are necessary which results in strong buoyancy effects and strong compensating air flows. The experiments have shown that the tracer gas propagates within few minutes to the upper parts of the stations.

The propagation paths unfortunately overlap with the escape routes of passengers in catastrophic circumstances.

The geometry of subway tunnels has a major influence, because differences in elevation drive the natural background air flow. To forecast the spreading of toxic gas in subway systems detailed climatological data is needed. The gas release inside a running subway coach can affect wide parts of stations and even following trains.

\section{Conclusion}

The new integrated analysis and measuring system opens new opportunities to conduct more complex field experiments with less effort. The measurement campaign consists of five tracer gas experiments. An amount of over 6000 syringes would be necessary to cover the same measuring time with a sample rate of only minute rather than two seconds using previous methods. Experimental costs are reduced to a tenth with the method covering in this paper.

Existing hand held measuring system which were mainly used for leak detection of high voltage switch gears are no substitute for the new sensor, because their measuring range is too coarse.

The new sensors can detect $\mathrm{SF}_{6}$ contamination accurate from $0.05 \mathrm{ppm}$ to $50 \mathrm{ppm}$, a very low detection limit is warranted due to the high greenhouse potential of $\mathrm{SF}_{6}$.

Results of the field experiments, as mentioned in this paper, can be coupled with numerical simulations [6] or with pedestrian simulations $[7,8]$ to assess subway systems in many safety questions.

\section{Acknowledgements}

Conducting Research in critical infrastructures like subway system is very difficult and only possible with the permission of the operator. Our research caused a lot of effort on the part of the operators. We are grateful that subway operators provide us their tunnels and stations. Concerning safety questions, we are very pleased that we find operators which have the courage to work with us on these issues.

\section{References}

[1] Barnett, A. (2012). Has Terror Gone to Ground? Institute for Operations Research and the Management Sciences, Phoenix.

[2] Kennedy, D. J., Stokes, A. W. \& Klinowski, W. G. (1987). Resolving Complex Mine Ventilation Problems With Multiple Tracer Gases. In Proceedings of the 3rd Mine Ventilation Symposium

[3] Pflitsch, A., Brüne, M., Ringeis, J. \& KillingHeinze, M. (2010). ORGAMIR - Development of a safety system for reaction of an event with emission of hazardous airborne substances - like a terror attack or fire - based on subway climatology. In A. Lönnermark \& H. Ingason (Eds.), Proceedings from the fourth International Symposium on Tunnel Safety and Security (Vol. 1, pp. 451-462). Borås, Sweden: SP Technical Research Institute of Sweden. 
[4] Potje-Kamloth, K. (2014). Finding the ideal escape and emergency route. Retrieved from http://www.imm.fraunhofer.de/content/dam/imm/ de/documents/pdfs/PD_MAusKat_final.pdf

[5] Spiegel, J., Brüne, M., Dering, N., Pflitsch, A., Qian Z., Agnew, B., Paliacin, R. \& Irving, M. (2014). Propagation of tracer gas in a subway station controlled by natural ventilation. Journal of Heat Island Institute International, Vol. 9, 103107. Retrieved from http://www.heat-island.jp/web_journal/Special_Iss ue_7JGM/S4_spiegel.pdf

[6] Spiegel, J., Letzel, M., Flassak, T. \& Pflitsch, A. (2014). Dispersion of Airborne Toxins in a highly complex Subway Station. In Proceedings of the 6th International Symposium on Tunnel Safety and Security (pp. 255-264). Marseille.

[7] Qian, Z., Agnew, B. \& Thompson, E. M. (2014). Simulation of Air flow, Smoke Dispersion and Evacuation of the Monument Metro Station based on Subway Climatology - Northumbria Research Link. Retrieved March 04, 2015, from Northumbria University: http://nrl.northumbria.ac.uk/21189/

[8] Brüne, M., Charlton, J., Pflitsch, A. \& Agnew, B. (2014). Coupling tracer gas experiments with evacuation simulation: An empirical approach to assess the effectiveness of evacuation routes in subway stations. In Proceedings of the 6th International Symposium on Tunnel Safety and Security (pp. 505-512). Marseille. 